\title{
Lagrangian transport of water vapor and cloud water in the ECHAM4 GCM and its impact on the cold bias
}

\author{
A. Stenke $\cdot$ V. Grewe $\cdot$ M. Ponater
}

Received: 12 December 2005/Accepted: 2 November 2007/Published online: 13 December 2007

(C) Springer-Verlag 2007

\begin{abstract}
The Lagrangian advection scheme ATTILA has been applied for the transport of water vapor and cloud water in the general circulation model (GCM) ECHAM4.L39(DLR) (E39) instead of the operational semi-Lagrangian transport scheme (SLT). ATTILA is a purely Lagrangian scheme that is numerically non-diffusive, while the operational semi-Lagrangian scheme exhibits a considerable numerical diffusion in the presence of sharp gradients. The model version E39/SLT significantly overestimates the water vapor mixing ratio in the extratropical lowermost stratosphere (wet bias) by a factor of 3-5 compared to HALOE observations. Compared to E39/SLT, E39/ATTILA shows substantially reduced water vapor mixing ratios in the extratropical lowermost stratosphere up to $70 \%$, and a steeper meridional water vapor gradient in the subtropics which is in better agreement with observations. Furthermore, the temperature distribution as simulated with E39/SLT is characterized by a pronounced cold temperature bias in the extratropical lowermost stratosphere (cold bias) and in the polar stratosphere above $50 \mathrm{hPa}$ in winter (cold pole). The improvements concerning the water vapor distribution in E39/ATTILA lead to a substantial reduction of the simulated cold bias by approximately 5-7 $\mathrm{K}$ which also results in a better representation of the modeled tropopause, especially in the extratropics. Sensitivity studies indicate that the warming of the extratropical lowermost stratosphere in E39/ATTILA is directly related to the reduced wet bias resulting in a less infrared radiative cooling. Additionally, the cold pole
\end{abstract}

A. Stenke $(\bowtie) \cdot$ V. Grewe $\cdot$ M. Ponater

Institut für Physik der Atmosphäre,

DLR-Oberpfaffenhofen, 82234 Wessling, Germany

e-mail: andrea.stenke@dlr.de problem is also slightly reduced in E39/ATTILA by approximately $2-5 \mathrm{~K}$.

\section{Introduction}

The correct representation of water vapor is a potential problem in general circulation models (GCMs) (Mote et al. 1994), since the water vapor distribution depends on a variety of physical processes on different temporal and spatial scales (e.g. Kley et al. 2000), some of them on unresolved scales. As shown in Fig. 1, the GCM ECHAM4.L39(DLR) (E39) exhibits a serious "wet bias" in the extratropical lowermost stratosphere, i.e. simulated water vapor mixing ratios are overestimated by a factor of 3-5 compared to HALOE observations. Due to the infrared absorption characteristics of water vapor (Clough et al. 1992), the simulated wet bias might strongly affect modeled temperatures and dynamics. In fact, this study will show that the simulated moist bias in the extratropical lowermost stratosphere contributes, due to its radiative effect, substantially to a systematic cold temperature bias in this region which is known as "cold bias" (Sect.5.2). The cold bias problem is present in many other atmospheric GCMs (e.g. Gates et al. 1999) and has shown little sensitivity to vertical (Land et al. 1999) and horizontal resolution (Stendel and Roeckner 1998). As for the lowermost stratosphere (in contrast to altitudes near $20 \mathrm{~km}$ ) it is also not eliminated by lifting the height of the model top and including an appropriate gravity wave drag scheme (e.g. Pawson et al. 2000).

In this paper we use the purely Lagrangian advection scheme ATTILA for the transport of water vapor and cloud water in the ECHAM4 GCM. The intention of this paper is 


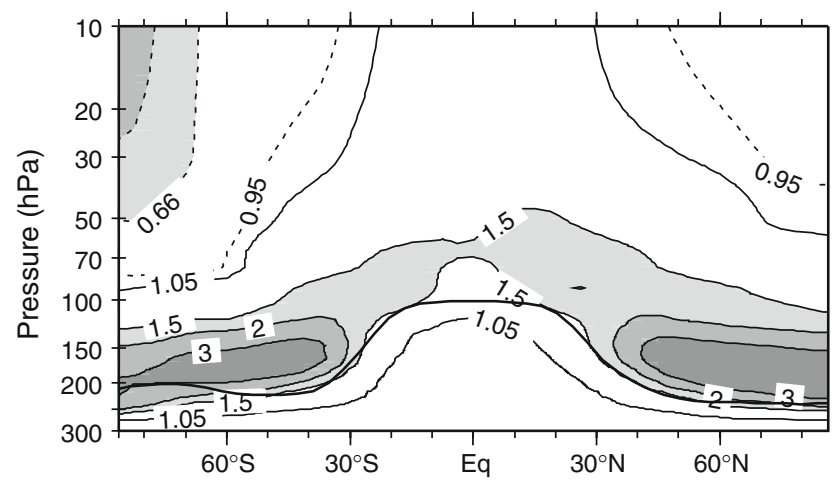

Fig. 1 Modeled "wet bias" in the extratropical lowermost stratosphere. The figure shows the ratio of the annually averaged zonal mean water vapor mixing ratio in E39/SLT to HALOE water vapor observations (provided by J.-U. Grooß, FZ Jülich). HALOE observations can be regarded to be useful only above the tropopause. The bold line indicates the simulated annually averaged zonal mean height of the thermal tropopause. Dashed contours indicate factors less than 1. Contours: $0.5,0.66,0.95,1.05,1.5,2,3$

to investigate the impact of the applied transport scheme on the modeled water vapor distribution in the upper troposphere/lower stratosphere (UTLS) region, and, furthermore, to discuss the impact of the modeled water vapor distribution on model dynamics, i.e. temperature and zonal wind.

Eulerian approaches, like the semi-Lagrangian advection scheme by Williamson and Rasch (1994) which is regularly used for the tracer transport in the ECHAM4 GCM, show a satisfactory performance in case of homogeneously distributed trace species, but they can lead to several problems when applied to highly inhomogeneous tracer distributions. In case of sharp gradients, many Eulerian as well as semi-Lagrangian schemes show a considerable numerical diffusion and tend to produce smooth tracer distributions. However, numerical diffusion seems to decrease with increasing horizontal and vertical model resolution (Rasch and Williamson 1990; Rasch and Lawrence 1998), i.e. when gradients are reasonably resolved. For example, Grewe et al. (2002) have shown that the numerical diffusion is significantly decreased in the 39 level version E39 compared to the standard ECHAM4 model with 19 vertical levels.

Lagrangian approaches, on the other hand, divide the model atmosphere into a large number of air parcels which are advected by the simulated wind field. The trace species are represented as mass mixing ratio on each parcel. Since such an advection scheme does not alter the tracer concentrations themselves, but the locations of air parcels carrying the concentration, it is numerically non-diffusive. Furthermore, Lagrangian advection itself is strictly mass conserving, while in semi-Lagrangian methods often a fixer has to be employed to adjust the total mass and guarantee conservation (Rasch and Williamson 1990). Such mass fixing produces artificial transport tendencies which may further cause an unfavorable smoothing of tracer distributions (e.g. Timmreck et al. 1999).

Transport studies with the ECHAM4 GCM using passive trace species like ${ }^{222} \mathrm{Rn}$ and ${ }^{14} \mathrm{CO}_{2}$ (Reithmeier and Sausen 2002) have shown that the purely Lagrangian transport scheme ATTILA is able to maintain steeper and more realistic gradients than the operational semi-Lagrangian scheme. ${ }^{222} \mathrm{Rn}$ exhibits a sharp meridional gradient at the subtropical tropopause, with high mixing ratios in the tropical upper troposphere and low mixing ratios in the extratropical lowermost stratosphere. Reithmeier and Sausen (2002) found that at the $100 \mathrm{hPa}$ level the semiLagrangian scheme produces much higher ${ }^{222} \mathrm{Rn}$ concentrations in extratropical regions than ATTILA. The authors attributed these differences to the enhanced horizontal diffusion across the subtropical tropopause in the semiLagrangian scheme. Water vapor also shows a strong meridional gradient between the tropical upper troposphere and the extratropical lowermost stratosphere, comparable to ${ }^{222} \mathrm{Rn}$. Therefore, the results of Reithmeier and Sausen (2002) suggest that the modeled wet bias might be caused by the numerical diffusion of the operational semiLagrangian advection scheme.

So far, ATTILA has been applied for the transport of passive tracers exclusively (Reithmeier and Sausen 2002). In a first step, ATTILA has been extended to treat the transport of water vapor and cloud water. In a second step, some free parameters in the cloud physics parameterization had to be re-adjusted in order to restore the long-term mean radiative balance at the top of the atmosphere.

In the following section a brief description of the GCM E39 and the Lagrangian transport scheme ATTILA is given. Necessary modifications to ATTILA associated with the Lagrangian transport of water vapor and cloud water are addressed in Sect. 3. Section 4 provides a short overview of the performed model experiments. In Sect. 5 the results of a model simulation with E39/ATTILA are compared with respective results of the standard model E39 and observations. A summary and concluding remarks are given in Sect. 6.

\section{Model description}

\subsection{The general circulation model ECHAM4.L39(DLR)}

The spectral general circulation model ECHAM4. L39(DLR) (hereafter E39, Land et al. 2002) is a derivate of the climate model ECHAM4 (Roeckner et al. 1996), with an enhanced vertical resolution of 39 levels up to the top layer centered at $10 \mathrm{hPa}$. ECHAM4 is based on the primitive equations with the prognostic variables vorticity, 
divergence, temperature, logarithm of surface pressure, humidity, cloud water, and, optionally, the mixing ratios of up to 21 passive tracers.

Water vapor, cloud water, and trace species are advected by the semi-Lagrangian transport (hereafter referred to as SLT) scheme of Williamson and Rasch (1994). Since the SLT scheme itself is not mass conserving, a mass fixer has to be applied which is an integral part of the SLT scheme in ECHAM4 (Rasch and Williamson 1990).

The model contains state-of-the-art parameterizations of radiation, cumulus convection, cloud formation and precipitation, horizontal diffusion, surface fluxes and vertical diffusion, orographic gravity wave drag and land surface processes (see Roeckner et al. 1996, and references therein). Compared to the standard model version, E39 includes a slightly modified formulation of the mass-flux convection scheme (Tiedtke 1989), which ensures strictly positive tracer concentrations (Brinkop and Sausen 1997).

A spectral horizontal resolution of $\mathrm{T} 30\left(\approx 6^{\circ}\right.$ isotropic resolution) is used in this study. The corresponding Gaussian transform grid, on which the semi-Lagrangian tracer transport and the model physics are calculated, has a mesh size of approximately $3.75^{\circ} \times 3.75^{\circ}$. The chosen time step is $30 \mathrm{~min}$.

\subsection{The Lagrangian transport scheme ATTILA}

ATTILA (Reithmeier and Sausen 2002) is a Lagrangian transport scheme which can be used as an alternative to the operational SLT scheme to transport passive tracers in the ECHAM4 GCM as well as in the ECHAM5 GCM (Roeckner et al. 2003) within the MESSy interface (Jöckel et al. 2005).

To cope with the applied spatial resolution (T30, 39 vertical levels) the model atmosphere is divided into about 498,000 air parcels of equal mass. At the initial time step the air parcels are distributed according to the mass of air in a grid box. Depending on the atmospheric region, the average number of air parcels per grid box ranges between 0.06 (top layer in polar regions) and 6 (at the equator at about $600 \mathrm{hPa}$ ). A distribution which is proportional to the air density $\rho$ is supposed to be stationary, since the mean mass flux divergence $\nabla \cdot(\rho \boldsymbol{u})$ is equal to zero in the ECHAM4 GCM. As shown by Reithmeier and Sausen (2002), the trajectories remain well distributed in the model atmosphere throughout the whole integration time. Therefore, no re-initialization of the air parcels during a model integration is necessary. At every time step the centroid of each air parcel is advected using the actual wind field. The trajectories of the air parcels are calculated three-dimensionally using the fourth order Runge-Kutta method. Winds are assessed by linear interpolation horizontally and by cubic Hermite interpolation vertically from the wind field on the ECHAM grid. After each advection step, air parcels located in the boundary layer are randomly re-located in the vertical. Thereby it is assumed that, after one time step, air parcels in the boundary layer will have "forgotten" their vertical position due to rapid turbulent mixing.

The mass mixing ratios of the trace species are defined on the centroids and representative for the whole air parcel. Strictly speaking, the air parcels are mass points without spatial extension, but they represent a certain volume of air. During advection the air parcels are considered to be isolated. However, in reality, the mixing among air parcels occurs. In ATTILA the issue of inter-parcel mixing is addressed by bringing the mass mixing ratio $c$ of a species in an air parcel closer to a background mixing ratio $\bar{c}$. For this purpose, the term $(\bar{c}-c) d$ is added to the mass mixing ratio $c$ at every time step. The background mixing ratio $\bar{c}$ is the average mixing ratio of all air parcels within a grid box. The rate of exchange is controlled by the dimensionless parameter $d$. Reithmeier and Sausen (2002) used a constant value of $d=10^{-3}$ in the troposphere, and of $d=5 \times 10^{-4}$ in the stratosphere, with the division between both regions being arbitrarily made at model level 12 (approximately $160 \mathrm{hPa}$ ). However, an alternative option in ATTILA is to calculate the parameter $d$ from the horizontal wind shear (Smagorinsky 1963):

$d=1-\frac{1}{1+2 k^{2} S \Delta t}$,

where $S$ is the horizontal wind shear, $\Delta t$ is the time step and $k=0.4$ is a diffusion constant. $d$ is calculated from the instantaneous local horizontal wind shear. The vertical wind shear is not considered. This approach is also used in the GRANTOUR model (Walton et al. 1988). Since sensitivity studies showed that calculating $d$ from the wind shear yields better results with regard to water vapor in the UTLS (Upper Troposphere/Lower Stratosphere), the latter method is used in this study for both water vapor and cloud water. However, it should be mentioned that the model results are not very sensitive to changes in $d$.

Convective events play an important role in tracer transport. ATTILA uses the ECHAM convection scheme by Tiedtke (1989) to include the vertical tracer transport due to subgrid-scale convection. For this purpose, the tracer concentrations are mapped from the air parcels to the ECHAM grid by calculating the average tracer concentration of all air parcels within a grid box at every time step. The concentrations on the model grid serve as input to the convection scheme. The convection scheme calculates mass fluxes from which the change of tracer mass in a grid box can be derived. The calculated tendencies are mapped back to the air parcels. In case of "empty grid boxes", i.e. grid boxes with no air parcels in them, the calculated 
convective mass fluxes to and from such a grid box are set to zero, which ensures zero tendency in that grid box and mass conservation, but also means an artificial reduction of convective transport. Since the occurrence of empty grid boxes is restricted to regions with less convective events, i.e. in the uppermost and lowermost model layers near the poles, this effect is small. It should be emphasized that the described calculation of convective mass fluxes is only valid for passive tracers. In case of water vapor and cloud water, some modifications have been necessary which are described in the next section.

Disadvantages concerning "empty grid boxes" are not caused by the Lagrangian approach itself, but result from inconsistencies regarding the representation of different physical processes like advection, convection or cloud physics. A fully Lagrangian approach could solve this problem. Currently a purely Lagrangian convection scheme is under development (S. Brinkop 2006, personal communication).

\section{Lagrangian water vapor transport}

So far, ATTILA has been used for the transport of passive tracers exclusively. The transport of water vapor and cloud water requires some extensions to ATTILA, e.g. concerning the representation of physics or empty gridboxes. The simulation of water vapor and cloud water is subdivided into two parts, the Lagrangian advection (left part of Fig. 2) and the parameterization of physical processes which are calculated in the Eulerian space (right part of Fig. 2).

For the calculation of physical processes the mass mixing ratios of water vapor and cloud water are mapped

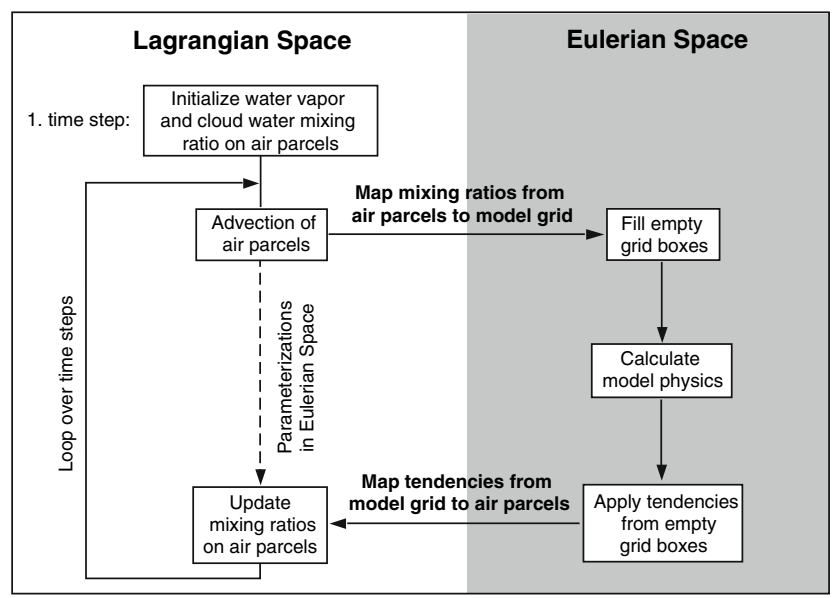

Fig. 2 Schematic overview of the Lagrangian transport of water vapor and cloud water, see text for details from the Lagrangian air parcels to the model grid by calculating the average mixing ratio of all air parcels within a certain grid box. In case of water vapor and cloud water, the calculation of radiation as well as convective and stratiform cloud formation and precipitation are performed on the model grid (right part of Fig. 2). Since the radiation scheme requires a complete humidity field, empty grid boxes have to be "filled", i.e. a reasonable water vapor mixing ratio has to be assigned to the empty grid box. In order to fill the empty grid boxes, a suitable algorithm has been introduced to search the nearest non-empty grid box, i.e. the nearest grid box containing at least one air parcel. The mixing ratio of the nearest non-empty grid box is assigned to the empty grid box. In case of cloud water empty grid boxes are not filled since cloud water shows a highly inhomogeneous distribution anyway and is not necessarily present in every grid box. After filling all empty grid boxes, the model physics are calculated. This filling routine makes the previously mentioned special treatment of convective mass fluxes from and to empty grid boxes unnecessary.

The physics routine returns tendencies for water vapor and cloud water, i.e. the change of mixing ratio in a grid box per time step due to the model physics. In order to transfer the total change of mass back into the Lagrangian space, tendencies calculated for empty grid boxes can not be ignored, but have to be attributed to air parcels. Again, the nearest non-empty grid box is determined and the tendency referred to the empty grid box is added. Furthermore, the tendencies may not be treated as change of mixing ratio, but as change of total mass of water vapor and cloud water within a grid box. Therefore, the mixing ratio tendencies are multiplied with the mass of air in the actual grid box to obtain total mass tendencies. This step is necessary to ensure mass conservation of the tendencies.

The calculated tendencies are mapped back to the air parcels. Positive tendencies are evenly distributed among the air parcels within a grid cell. Negative tendencies, i.e. the grid volume loses water vapor or cloud water, are distributed proportional to the available tracer mass on each parcel to avoid negative mixing ratios on the air parcels. After that, the total mass tendencies are transformed back into mixing ratio tendencies, i.e. (change of tracer mass in air parcel)/(mass of air parcel)/timestep, and the mixing ratios on the air parcels are updated by applying the respective tendencies.

We have emphasized in the introduction that one of the desirable properties of transport schemes is the conservation of mass. The shortcomings of the semi-Lagrangian scheme in this respect are quite obvious, but also in the Lagrangian scheme some compromises are necessary. The Lagrangian advection of water vapor and cloud water itself is strictly mass conserving, since only the locations of the 
air parcels carrying the tracer concentration are affected, but not the tracer concentrations themselves. However, the mapping from the Lagrangian air parcels to the model grid is not mass conserving, since the density distributions in the Lagrangian and in the Eulerian space differ, i.e. the air mass within a grid box does not necessarily equal the sum of the masses of all air parcels within that box. In our simulation the relative error in the globally integrated mass of water vapor between Lagrangian and Eulerian space was about $1 \%$. Note that this error does not influence the tracer mass in the Lagrangian space, i.e. it does not propagate or accumulate. A solution to this problem would be a fully Lagrangian kernel ensuring a consistent density distribution, but this is beyond the scope of this study. Furthermore, the transformation of the Eulerian tendencies back to the Lagrangian space is mass conserving in a sense that the tendencies used for updating the concentrations on the air parcels equal the change of mass in the Eulerian space. However, in ECHAM4 the physical parameterizations of the hydrological cycle themselves are not mass conserving, independent of the choice of the advection scheme. The global annual mean evaporation and precipitation show a small imbalance of about $0.4 \%$, partly due to subgrid-scale cumulus convection (Roeckner et al. 1996). Hence, in view of global mass conservation, it has to be distinguished between Lagrangian advection conservation, the mapping from Lagrangian to Eulerian space, and the treatment of physical parameterizations.

\section{Experimental set-up}

In a first step, ATTILA has been modified to treat the Lagrangian transport of water vapor and cloud water as described above. The results of a pilot simulation have shown that the water vapor and cloud water distribution as simulated with E39/ATTILA affects the modeled cloud cover and therefore the climatological mean radiation budget at the top of the atmosphere. Many parameterization schemes, like the stratiform cloud scheme, contain some model-dependent parameters, whose values are not exactly known. These values may be adjusted to reach an optimal performance of the scheme, and they might have to be re-adjusted if conditions outside the parameterization, like the water vapor distribution, change.

In case of the pilot experiment the induced radiative imbalance amounts to approximately $10 \mathrm{~W} \mathrm{~m}^{-2}$ in the global annual average. This experiment does not allow a fair comparison of the simulated parameters to the welltuned reference simulation with E39/SLT. In order to reduce the overestimated cloud cover of the pilot simulation and to restore the climatological global mean balance of incoming solar and outgoing longwave radiation at the top of the atmosphere, the following model parameters have been re-adjusted in a second step: The threshold relative humidity, which controls the onset of cloud formation at higher levels (Xu and Krüger 1991), was increased from 0.6 to 0.7 . Thus cloud formation is impeded. In order to reduce the lifetime of water clouds the efficiency of stratiform rain formation was enhanced by increasing the coalescence rate. In case of convective precipitation the coefficient controlling the conversion rate of cloud droplets to rain drops was re-adjusted to a higher value. Finally, the efficiency of sedimentation of ice crystals (Heymsfield 1977) was enhanced, i.e. the lifetime of ice clouds is also reduced compared to the standard version of E39 using the semi-Lagrangian advection scheme (hereafter referred to as E39/SLT). A similar re-adjustment of model parameters had to be performed after changing the vertical resolution of the ECHAM4 model from 19 to 39 levels (Land et al. 1999, 2002).

The impact of the numerical properties of the applied advection scheme on the water vapor distribution is investigated on the basis of two multiyear model simulations with E39/ATTILA and E39/SLT. After a spin-up time of 10 years both model versions have been integrated over 10 annual cycles in a quasi-equilibrium mode representing atmospheric conditions of the year 1990 (time slice simulation).

\section{Results}

In this section the distributions of water vapor and cloud water as well as temperature and zonal wind as simulated with the above described model version E39/ATTILA are presented. Furthermore, the model results are compared with respective results from the standard version E39/SLT as well as with observations or ECMWF re-analyses data from 1985 to 1995 (ERA40) (Uppala et al. 2005).

\subsection{Water vapor and cloud water}

Figure 3 displays the zonal mean water vapor mixing ratio for the standard model E39/SLT (a) and the model version E39/ ATTILA (b). In order to allow a better comparison between both model versions in the most sensitive region around the tropopause, the differences (E39/ATTILA minus E39/SLT) above $500 \mathrm{hPa}$ are shown in Fig. 3c. Both model versions reproduce the observed strong vertical gradient in the troposphere following the temperature gradient. The lowest water vapor mixing ratios occur just above the tropical tropopause, where temperatures are low, and in the southern hemisphere polar regions during winter associated with dehydration in the Antarctic polar vortex. It should be mentioned that a source of 
Fig. 3 Climatological averages of the zonal mean water vapor mixing ratio (ppmv) for January and July: a E39/SLT, b E39/ ATTILA, c difference E39/ ATTILA-E39/SLT. Light (dark) shaded areas indicate the 95\% (99\%) significance level of the displayed differences $(t$ test). The bold line indicates the monthly averaged zonal mean tropopause height for each model version. In the lower panel (c) the zonal mean tropopause as simulated with E39/ATTILA (b) is shown a)
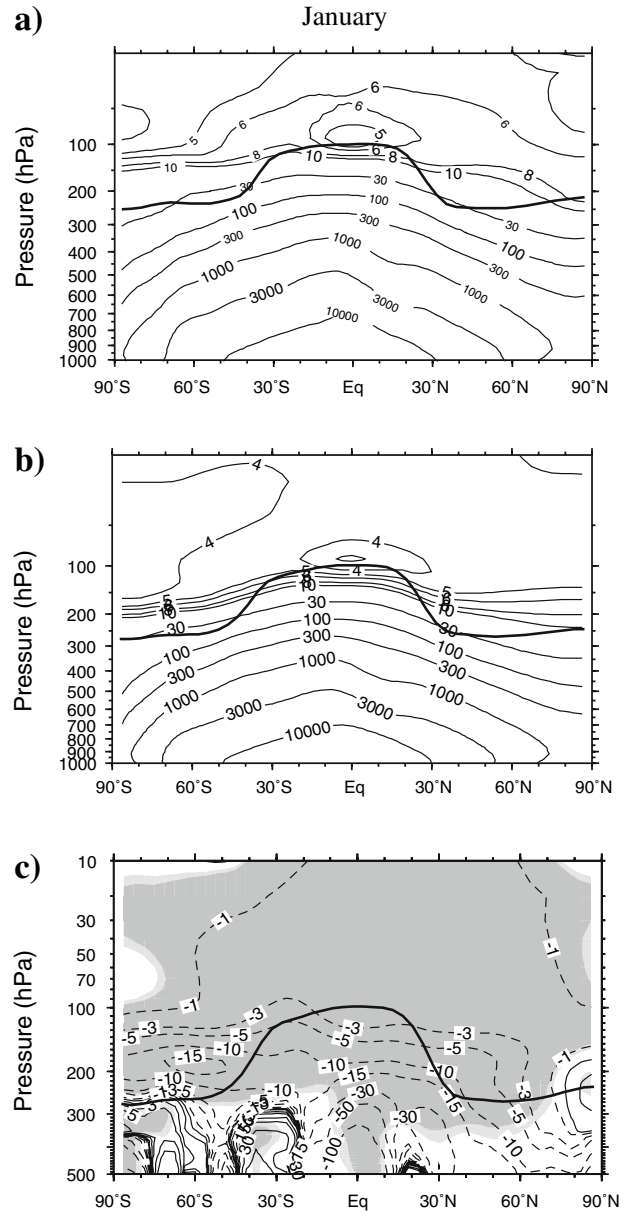
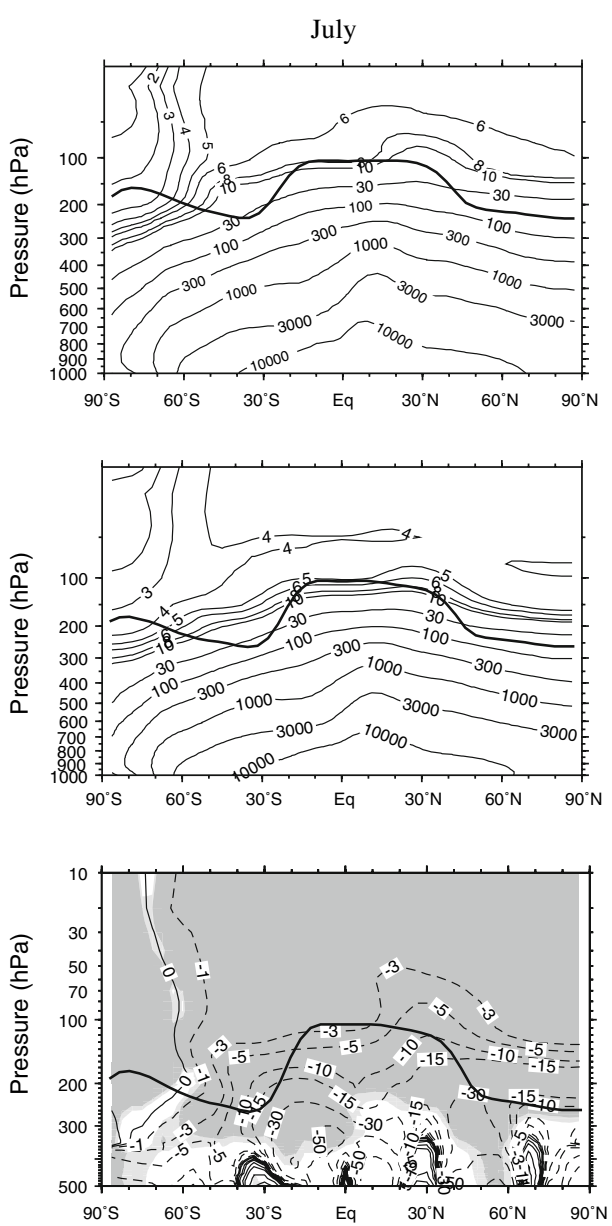

stratospheric water vapor from methane oxidation is missing in both model versions. Therefore, the observed water vapor increase upward and poleward, which is associated with the downward transport of moist air masses from higher altitudes via the Brewer-Dobson-circulation, is not reproduced. This leads to an underestimation of water vapor mixing ratios at higher latitudes above $70 \mathrm{hPa}$ compared to HALOE observations (Fig. 1).

In the troposphere, the simulated water vapor distribution is similar for both models E39/SLT and E39/ATTILA. Differences in the lower and middle troposphere are below 10\%. The most pronounced deviations between E39/SLT and E39/ATTILA occur in the extratropical lowermost stratosphere and in the southern hemisphere polar stratosphere during winter (Fig. 3c). E39/ATTILA simulates significantly lower water vapor mixing ratios in the extratropical lowermost stratosphere compared to E39/SLT. Maximum differences of -15 to $-30 \mathrm{ppmv}$ are found between 150 and $250 \mathrm{hPa}$ in the respective summer hemisphere. These values correspond to a water vapor reduction of about $70 \%$. This implies that E39/ATTILA is able to retain a stronger meridional water vapor gradient in the subtropical UTLS region compared to E39/SLT. Above the lowermost stratosphere, i.e. at levels higher than about $100 \mathrm{hPa}$ water vapor mixing ratios are generally about 1-3 ppmv lower in E39/ATTILA than in E39/SLT, except for the Antarctic winter stratosphere. The overall reduction in stratospheric water vapor is a major improvement compared to E39/SLT model versions which showed a stratospheric water vapor excess of approximately 2-3 ppmv compared to observations (Stenke and Grewe 2005). Though there is still an ongoing debate about the different processes governing the stratospheric water vapor distribution, it is agreed on that the water vapor content of air masses entering the stratosphere is controlled by the temperatures near the tropical tropopause. Therefore, this overall reduction in stratospheric water vapor is most likely associated with a change in tropical tropopause temperatures and, hence, in the saturation mixing ratio (see Sect.5.2). Table 1 summarizes the annual mean changes of water vapor mass in the extratropical lowermost stratosphere and above a reference level of $70 \mathrm{hPa}$. In this context, the extratropical lowermost stratosphere denotes the region polewards of $40^{\circ} \mathrm{N} / \mathrm{S}$ between 120 and $220 \mathrm{hPa}$.

Dehydration of the Antarctic polar vortex in winter is overestimated in E39/SLT compared with observations. 
Table 1 Annual mean water vapor mass in the extratropical lowermost stratosphere (LMS) and above $70 \mathrm{hPa}$ for E39/SLT and E39/ ATTILA

\begin{tabular}{llll}
\hline & \multicolumn{2}{l}{$\mathrm{H}_{2} \mathrm{O}(\mathrm{kg}), \mathrm{LMS}$} & $\begin{array}{l}\mathrm{H}_{2} \mathrm{O}(\mathrm{kg}) \\
\text { y }\end{array}$ \\
\cline { 2 - 3 } & $\mathrm{SH}$ & $\mathrm{NH}$ & above $70 \mathrm{hPa}$ \\
\hline E39/SLT & $7.5 \times 10^{11}$ & $10.8 \times 10^{11}$ & $11.4 \times 10^{11}$ \\
E39/ATTILA & $4.3 \times 10^{11}$ & $5.8 \times 10^{11}$ & $8.5 \times 10^{11}$ \\
\hline
\end{tabular}

The LMS value describes the water vapor mass polewards of $40^{\circ}$ between 120 and $220 \mathrm{hPa}$

$\mathrm{NH}$ northern hemisphere, $\mathrm{SH}$ southern hemisphere

This feature has been recognized also in model versions that have been coupled to a chemistry module (Hein et al. 2001; Dameris et al. 2005) and that include methane oxidation. Minimum water vapor mixing ratios below $1 \mathrm{ppmv}$ can be widely found in the Antarctic stratosphere above $70 \mathrm{hPa}$. This model behavior is directly related to the cold bias in the Antarctic polar stratospheric temperature (see Sect.5.2, Fig. 8a). In E39/ATTILA, the dehydration of the southern hemisphere polar stratosphere is less pronounced compared to E39/SLT, resulting in higher water vapor mixing ratios by about 1-2 ppmv which means a better agreement with observations (e.g. Grooß and Russell 2005). This water vapor increase can be directly attributed to changes in polar stratospheric temperatures (see Sect.5.2). The described water vapor changes are statistically significant almost everywhere, as indicated by the shaded areas.

The above discussed changes of the simulated water vapor distribution cause some remarkable effects on model dynamics, e.g. the tropopause height. As already indicated from Fig. 3, the modeled extratropical tropopause is approximately $700 \mathrm{~m}$ lower in E39/ATTILA than in E39/ SLT. The changes of modeled temperatures and tropopause characteristics will be discussed in detail in Sect. 5.2.

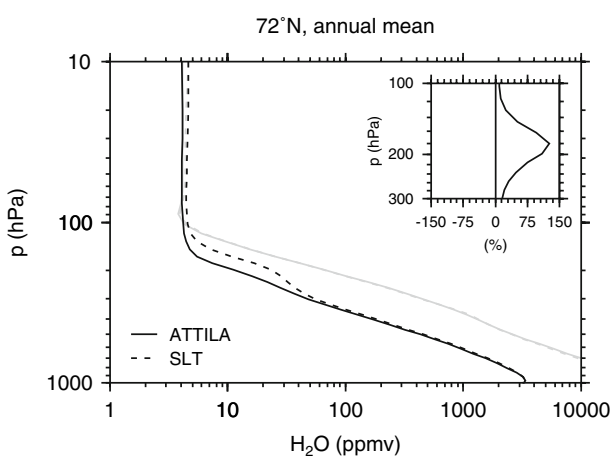

Fig. 4 Zonally and annually averaged profile of the water vapor mixing ratio as advected by ATTILA (solid) and the idealized water vapor tracer as advected by the semi-Lagrangian scheme (SLT dashed) at $72^{\circ} \mathrm{N}$ (left) and $72^{\circ} \mathrm{S}$ (right), respectively. The grey lines
The above presented results are based on simulations with the re-adjusted version of the GCM E39/ATTILA, i.e. after restoring the climatological mean radiation budget (see Sect. 4). Water vapor is radiatively active, i.e. changes in the water vapor distribution feed back to model dynamics which in turn affect the water vapor transport. Therefore, several factors like the re-adjustment of the cloud parameterization, changes in model dynamics as well as the numerical characteristics of the advection scheme may contribute in different extent to the improved water vapor distribution in E39/ATTILA. In order to separate the numerical effects of the applied advection scheme, we have performed a sensitivity simulation with E39/ATTILA including an idealized "water vapor" tracer which mimics the physical behavior of water vapor (convection and condensation) in this model, but is advected by the semiLagrangian transport scheme and does not feed back to model dynamics. Since both water vapor fields experience the same meteorological conditions in terms of wind field and temperature, the resulting differences can be directly attributed to the advection scheme. The zonally averaged profiles of water vapor (ATTILA) and the idealized tracer (SLT) at $72^{\circ} \mathrm{N} / \mathrm{S}$ are depicted in Fig. 4. The idealized SLTtracer shows a substantial moistening of the lowermost stratosphere caused by the semi-Lagrangian scheme. At this latitude the mixing ratio of the SLT-tracer is more than twice as large as the water vapor content in ATTILA. Above $100 \mathrm{hPa}$ the SLT-tracer still exceeds the water vapor content (ATTILA) by about 1-2 ppmv. In the troposphere, both profiles differ only marginally. In tropical regions (grey lines in Fig. 4), both water vapor profiles are almost identical throughout the troposphere, while in the stratosphere the SLT-tracer is slightly higher.

Since both schemes see the same meteorology, numerical issues have to be responsible for the described deviations between the SLT-tracer and the ATTILA water vapor. The tropical profiles indicate a vertical diffusion of water vapor

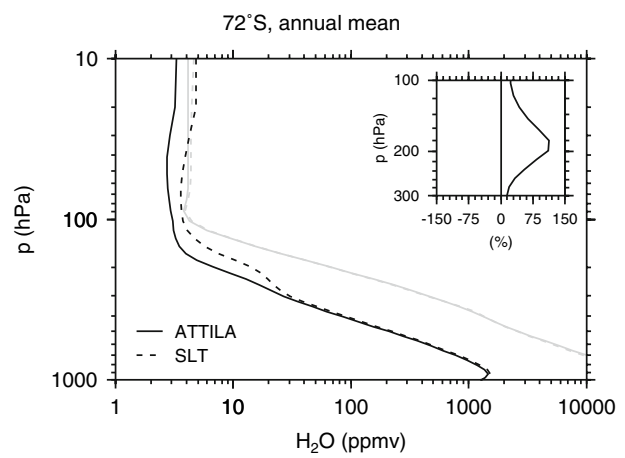

indicate the respective profiles at the Equator. The inserted figure shows the relative difference (SLT-ATTILA)/ATTILA (\%) for the region $100-300 \mathrm{hPa}$ 
from the troposphere into the stratosphere in the SLTscheme. This vertical diffusion is not necessarily restricted to tropical regions, but seems to be apparent at all latitudes. In the southern hemisphere, where the stratospheric water vapor mixing ratios are generally lower than in the northern hemisphere, the vertical gradient at the tropopause and, therefore, also the vertical diffusion are more pronounced. The deviations in the extratropical lowermost stratosphere $(100-250 \mathrm{hPa})$ are substantially larger than above the $100 \mathrm{hPa}$ level, and can not only be explained by vertical diffusion. Rather, meridional diffusion from the tropical upper troposphere into the extratropical lowermost stratosphere in the SLT scheme is the dominant process here which confirms the findings of Reithmeier and Sausen (2002) for passive tracers. The semi-Lagrangian scheme affects the distribution of the idealized "water vapor" tracer in the extratropical lowermost stratosphere in a similar way as shown in Fig. 3. This adds support to the notion that the considerable numerical diffusion of the semi-Lagrangian advection scheme is the main contributor to the simulated wet bias in E39/SLT.

Finally, Fig. 5 displays the ratio of the annually averaged zonal mean water vapor mixing ratio in E39/ATTILA to HALOE water vapor observations (see also Fig. 1). In contrast to E39/SLT, the water vapor mixing ratio in the extratropical lowermost stratosphere as simulated with E39/ATTILA is in good agreement with observations. However, in the extratropical stratosphere above $50 \mathrm{hPa}$ water vapor mixing ratios in E39/ATTILA are underestimated as in E39/SLT (see Fig. 1). As mentioned above, this behavior is directly related to the missing water vapor source from methane oxidation.

In order to evaluate the transport characteristics of E39/ ATTILA with respect to cloud water, Fig. 6 presents the zonal mean cloud water distribution (upper panel) and the zonal mean fractional cloud cover (lower panel) as

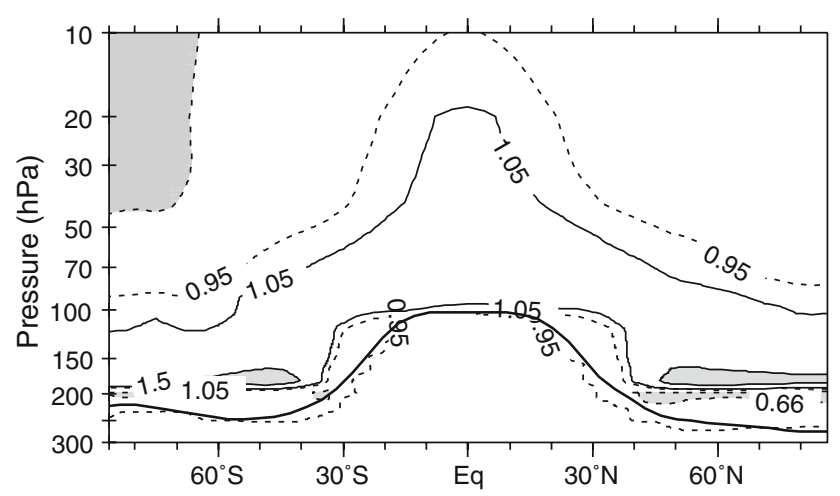

Fig. 5 Same as Fig. 1, but for E39/ATTILA and HALOE observations. The bold line indicates the simulated annually averaged zonal mean height of the thermal tropopause. Dashed contours indicate factors less than 1. Contours: 0.5, 0.66, 0.95, 1.05, 1.5, 2, 3 simulated with E39/SLT (left) and E39/ATTILA (right). The simulated cloud water distribution is very similar in both models, with maximum values in the lower troposphere in the tropics and the mid-latitudes of both hemispheres. Both model versions show a decreasing cloud water content with increasing latitude and altitude. Lower cloud water values are found in the subtropics, where subsiding air prevails.

Directly linked to the cloud water distribution is the simulated cloud cover which is shown in the lower panel of Fig. 6. Again both models agree in the main characteristics of cloud coverage, i.e. tropical maxima in the upper troposphere, high cloud amounts in lower levels at mid and polar latitudes and low cloudiness in subtropical regions. With respect to model differences E39/ATTILA shows an overall reduction of extratropical cloud coverage between 200 and $100 \mathrm{hPa}$ which can be attributed to a declining tropopause in E39/ATTILA relative to E39/ SLT (see Sect.5.2, Fig. 10). The high cloud cover in the southern polar stratosphere must be regarded as an artefact of an, for stratospheric conditions, inadequate cloud parameterization. However, as these clouds contain almost no ice water, their impact on the radiation budget is negligible (Land et al. 1999). Based on Fig. 6 we conclude that E39/ATTILA performs also well with respect to cloud water.

For a further evaluation of the modeled hydrological cycle the simulated geographical distribution of annual mean precipitation (Fig. 7) is compared with an observational precipitation climatology from 1990 to 1999 obtained within the Global Precipitation Climatology Project [GPCP version 2 combined precipitation data set, Adler et al. (2003)]. Both model versions reproduce the main characteristics of the observed precipitation distribution, i.e. small precipitation in polar regions, over the tropical oceans to the west of the continents, over the northern part of Africa, and large precipitation in the region of the intertropical convergence zone (ITCZ), at the west coast of North America. In the equatorial Indian Ocean both model versions simulate more precipitation than observed. The precipitation maximum over the northern Pacific is underestimated in E39/SLT, but E39/ATTILA shows a good agreement with GPCP. However, E39/ATTILA slightly overestimates precipitation in northern polar regions. The "oceanic deserts" in subtropical regions with less than $1 \mathrm{~mm} /$ day precipitation are smaller in the model simulations, especially in E39/ATTILA. The global mean precipitation amounts to $2.6 \mathrm{~mm} /$ day for GPCP, $2.8 \mathrm{~mm} /$ day for E39/SLT and $2.9 \mathrm{~mm} /$ day for E39/ATTILA, i.e. both model versions overestimate the observations by approximately $10 \%$. This comparison shows that the performance of E39/ATTILA with respect to precipitation is as good as of E39/SLT. 
Fig. 6 Annual averages of the zonal mean cloud water content $\left(10^{-6} \mathrm{~kg} / \mathrm{kg}\right.$, upper panel) and zonal mean fractional cloud cover (\%, lower panel). E39/ SLT (left) and E39/ATTILA (right)
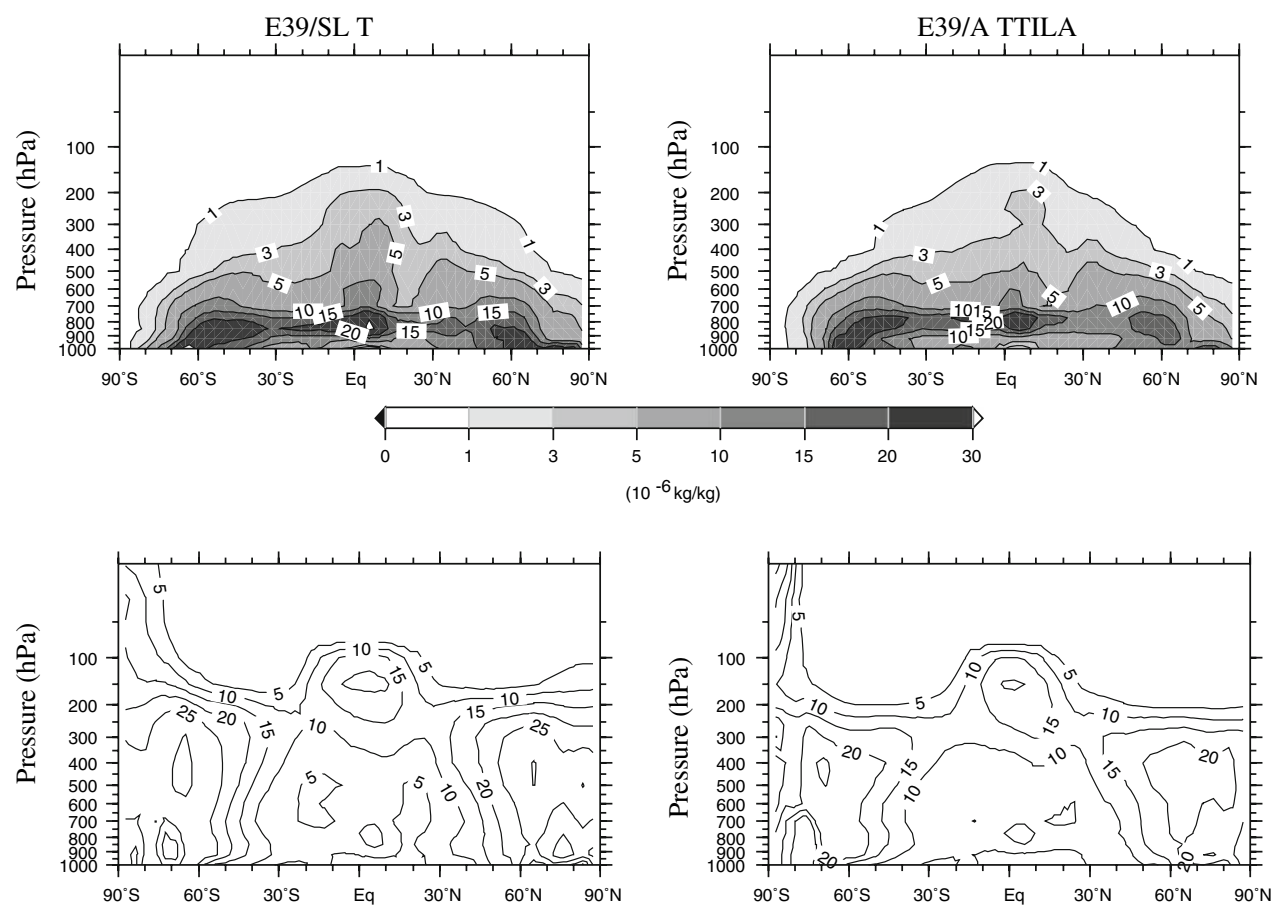

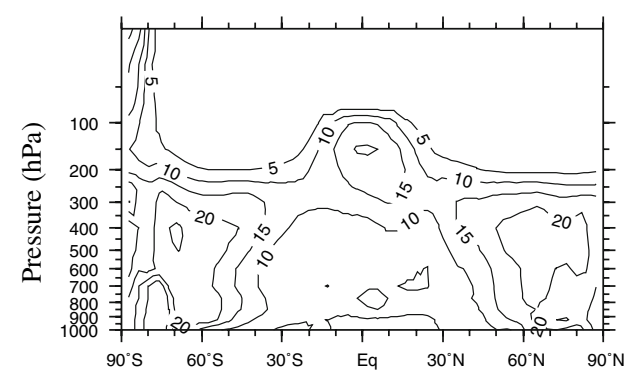

\subsection{Zonal mean temperature}

In this subsection, we investigate the impact of the modeled water vapor distribution on model dynamics. Here, we concentrate on the modeled zonal mean temperature distribution. The modeled tropopause characteristics and the zonally averaged zonal wind are presented in the following sections. The simulated zonal mean temperature distribution is additionally evaluated using ERA40 data.

Figure 8 shows the differences of modeled zonal mean temperatures and observations (ERA40, a) for both model versions E39/SLT (b) and E39/ATTILA (c). In the troposphere, the temperature deviations are small (1-2 K) for both model versions. In the tropical UTLS region, the temperature deviations show a dipole structure with a too warm upper troposphere and a too cold lower stratosphere for both model versions, resulting in a slightly too high tropical tropopause in both models (Fig. 10). The warm error in tropical upper tropospheric temperatures as well as the cold error in tropical lower stratospheric temperatures are less pronounced in E39/ATTILA compared to E39/ SLT. The largest temperature deviations in E39/SLT (Fig. 8b) occur in the lowermost polar stratosphere. A cold bias of more than $7 \mathrm{~K}$ can be found poleward of $60^{\circ} \mathrm{N} / \mathrm{S}$ at $200 \mathrm{hPa}$. As shown in Fig. 8c, the reduction of the simulated wet bias in the extratropical lowermost stratosphere results in a significant reduction of the modeled cold bias in E39/ATTILA: In the southern (northern) hemisphere, the temperature deviation now amounts approximately $-4 \mathrm{~K}$ $(-3 \mathrm{~K})$ which corresponds to a reduction of the cold bias of more than 50\% ("Cold Bias" in Table 2).
E39/SLT also simulates too low temperatures in polar regions of the respective winter hemisphere above $30 \mathrm{hPa}$ (referred to as "cold pole" problem), with a cold temperature bias of more than $17 \mathrm{~K}$ at the model top. The cold pole problem is still visible in the annual mean temperature distribution, especially in the southern hemisphere (Fig. 8b). As evident from Fig. 8c, this part of the cold pole problem is also slightly reduced in E39/ATTILA: In the southern hemisphere polar stratosphere modeled temperatures are approximately $16 \mathrm{~K}$ lower than ERA40 and the northern hemisphere modeled temperatures are $2.4 \mathrm{~K}$ lower ("Cold Pole" in Table 2). It should be mentioned that the ERA40 temperature climatology of the upper stratosphere in general, but especially over Antarctica, is still under investigation (Uppala et al. 2005). Therefore, temperature deviations near the E39 model top have to be interpreted carefully.

The above mentioned temperature differences between E39/ATTILA and E39/SLT include all direct and indirect effects of the water vapor change induced by the different transport scheme. In order to quantify the purely radiative effect of the simulated wet bias, several sensitivity simulations have been performed in which the model's radiation code was forced by a HALOE stratospheric water vapor climatology (provided by J.-U. Grooß, FZ Jülich) instead of its simulated values. The so-called stratospheric temperature adjustment (e.g. Stuber et al. 2001), which indicates the temperature response under the constraint of fixed dynamical heating rates (FDH approximation, Fels et al. 1980), has been applied to examine the radiative effect of the simulated wet bias. As known from previous 
a)

GPCP

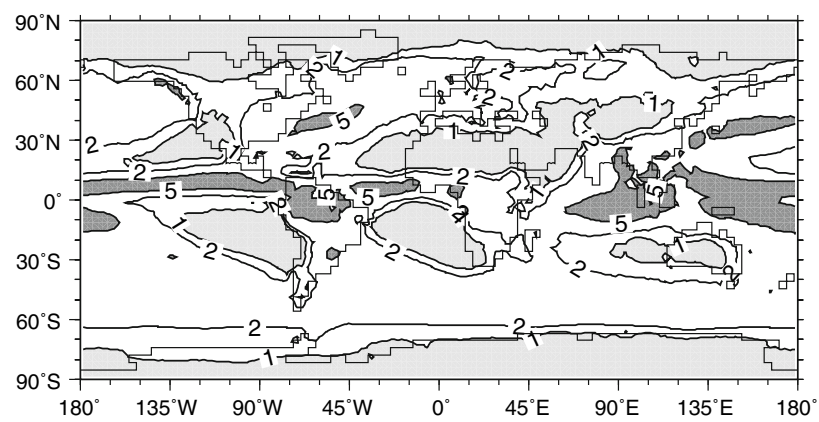

b)

E39/SLT

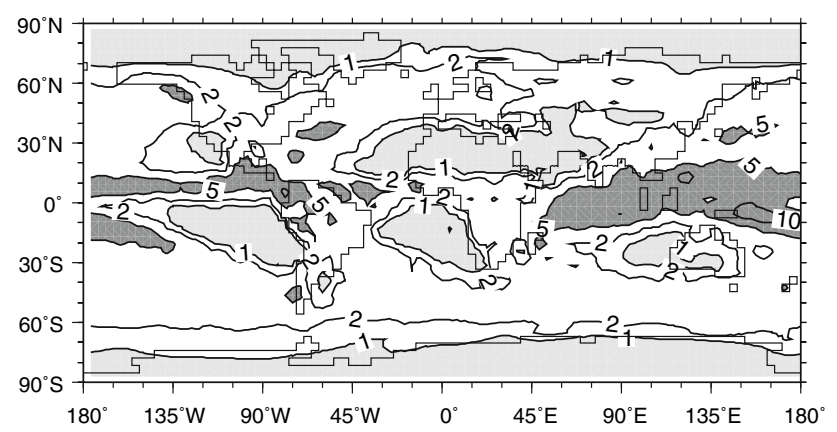

c)

E39/ATTILA

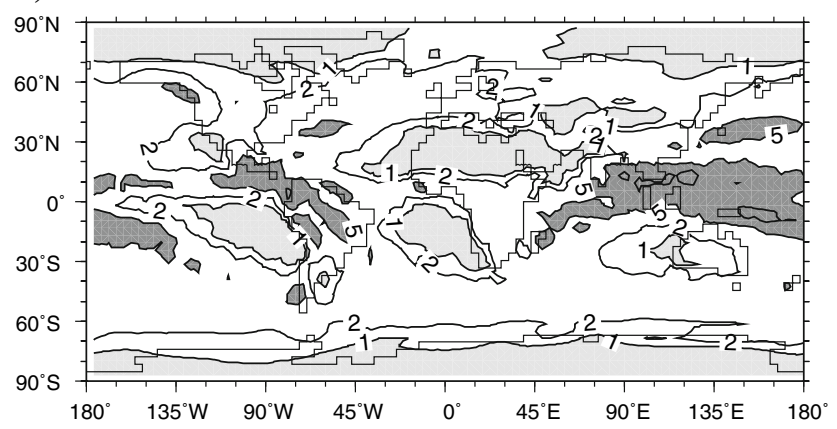

Fig. 7 Annual mean precipitation: a GPCP version 2 combined precipitation data set, b E39/SLT, c E39/ATTILA. Contour spacing is 1, 2, 5, 10, 15, $20 \mathrm{~mm} /$ day. Light (dark)shading indicates areas with $P<1(P>5) \mathrm{mm} / \mathrm{day}$

studies (e.g. Rosier and Shine 2000), the FDH approximation provides a very reasonable first order representation of the "full" response that includes all dynamics and physics feedbacks. Moreover, compared to simulations of the full model it allows to separate the direct radiative impact on the temperature field from the effect of the feedbacks. Figure 9a shows the temperature response for the FDH approximation. The results indicate a warming of 4-6 K in the extratropical lowermost stratosphere, reducing the simulated cold bias to about half of its original value. Figure $9 \mathrm{~b}$ presents the "full" temperature response, where additional heating rate changes from dynamical feedbacks contribute to a slightly different net temperature change. A comparison of Fig. 9a and b suggests that the

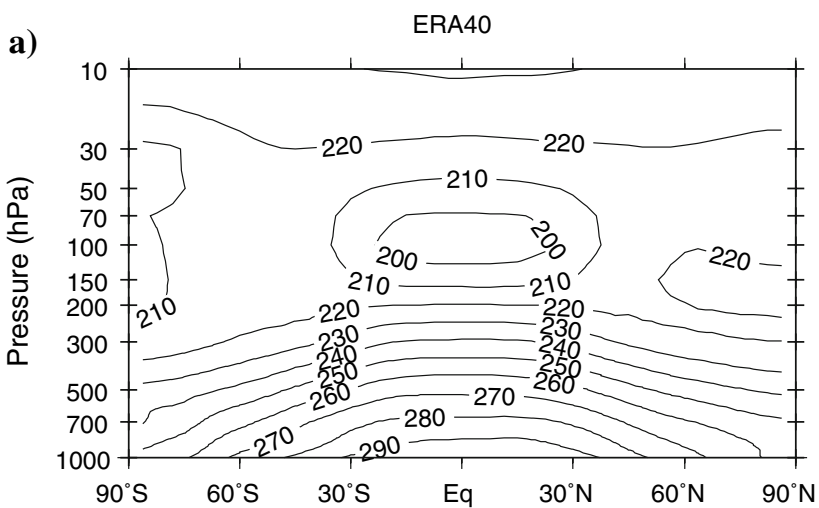

b) $\quad$ E39/SLT - ERA40

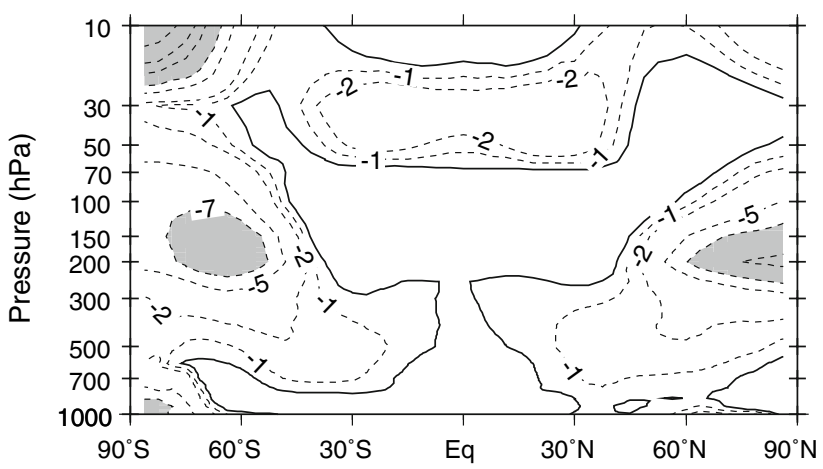

c)

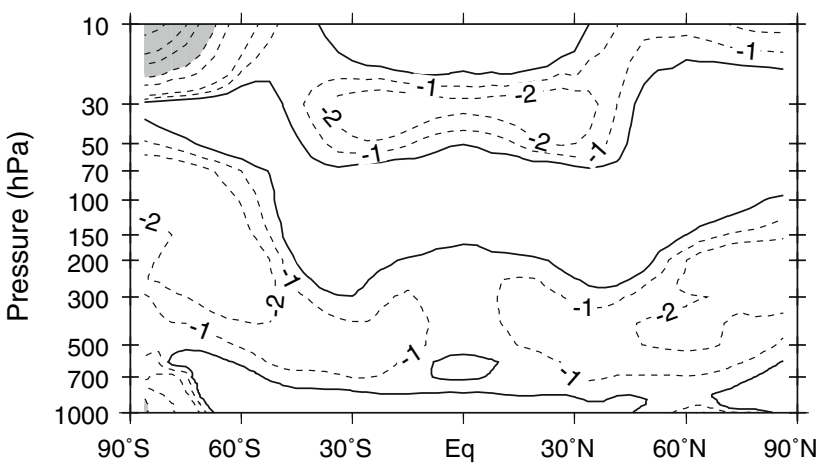

Fig. 8 ERA40 annual and zonal mean temperatures (a) and differences of modeled temperatures and ERA40 reanalyses: b E39/SLT-ERA40, c E39/ATTILA-ERA40. Contour lines are 0 (bold), $\pm 1, \pm 2, \pm 5$, $\pm 7, \pm 10, \pm 12$, and $\pm 15 \mathrm{~K}$, negative contours are dashed. Temperature differences greater than $7 \mathrm{~K}$ are shaded

temperature response to the prescribed water vapor distribution is not essentially modified by dynamical feedbacks. The direct radiative warming in the southern lowermost stratosphere is slightly reduced in subtropical latitudes while it is somewhat enhanced in polar regions. Generally, the results of the HALOE sensitivity experiments presented in Fig. 9 make it clear that the correlation between the wet and cold bias in fact indicates a forcing of the larger part of the cold bias by the radiative impact of the simulated water vapor excess. 
Table 2 Deviations of modeled temperatures, height of the extratropical thermal tropopause (TP) and strength of the polar night jet from ECMWF re-analyses (ERA40)

\begin{tabular}{|c|c|c|c|c|c|c|c|c|}
\hline & \multicolumn{2}{|c|}{ Cold Bias (K) } & \multicolumn{2}{|c|}{ Cold Pole (K) } & \multicolumn{2}{|c|}{ Extratrop. TP $(\mathrm{hPa})$} & \multicolumn{2}{|c|}{ Polar Night Jet $\left(\mathrm{m} \mathrm{s}^{-1}\right)$} \\
\hline & $\mathrm{SH}$ & $\mathrm{NH}$ & SH & NH & SH & NH & SH (Jul) & $\mathrm{NH}(\mathrm{Jan})$ \\
\hline E39/SLT & -8.9 & -9.1 & -17.6 & -4.0 & -35.8 & -44.2 & +16.1 & +10.7 \\
\hline E39/ATTILA & -4.3 & -3.4 & -16.1 & -2.4 & -14.3 & -14.5 & +10.9 & +12.7 \\
\hline
\end{tabular}

"Cold Bias": annual mean temperature at $200 \mathrm{hPa}, 70^{\circ} \mathrm{N} / \mathrm{S}$; "Cold Pole": annual mean temperature at $10 \mathrm{hPa}, 83^{\circ} \mathrm{N} / \mathrm{S}$; "Extratropical Tropopause": annual mean height of thermal tropopause polewards of $60^{\circ} \mathrm{N} / \mathrm{S}$; "Polar Night Jet": zonal wind speed at $10 \mathrm{hPa}, 60^{\circ} \mathrm{N}(\mathrm{January})$ and $60^{\circ} \mathrm{S}$ (July)

$\mathrm{NH}$ northern hemisphere value, $\mathrm{SH}$ southern hemisphere value
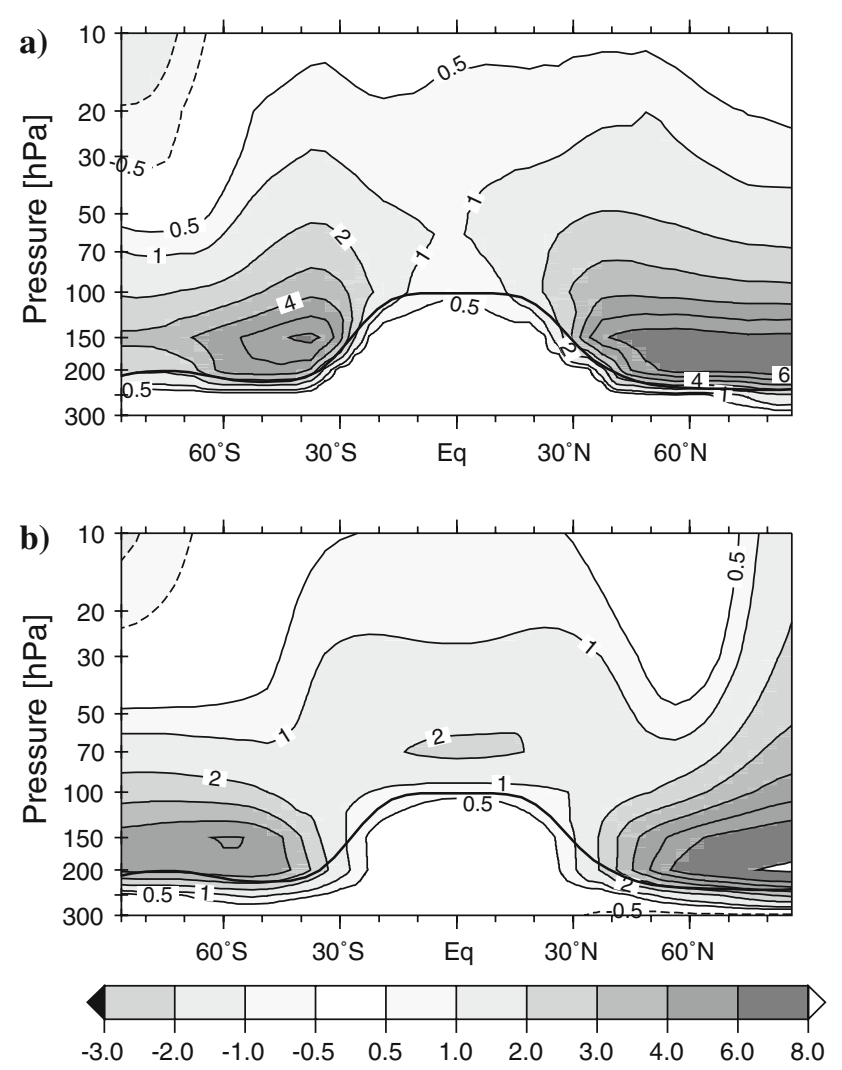

$(\mathrm{K})$

Fig. 9 Annual and zonal mean temperature response $(\mathrm{K})$ as simulated with E39/SLT using the HALOE water vapor climatology instead of the simulated water vapor as input for the calculation of radiative heating rates. a Fixed dynamical heating (FDH) approximation. b Full temperature response including dynamics and physics feedbacks. The bold line indicates the annually averaged zonal mean height of the thermal tropopause in E39/SLT

\subsection{Tropopause height}

The described improvements in modeled temperatures cause some remarkable changes of the simulated tropopause characteristics in E39/ATTILA. As shown in Fig. 10a, the modeled tropopause pressure (thermal tropopause, TP, calculated according to the method of Reichler et al. 2003) in polar regions in E39/SLT is approximately 40-60 hPa lower than observed (ERA40, bold dashed-dotted line in Fig. 10a). Compared to E39/ SLT, the tropopause pressure is about $20-30 \mathrm{hPa}$ higher in E39/ATTILA which means a much better representation of the extratropical tropopause (Table 2). In the tropics, both model versions simulate a slightly too high thermal tropopause, whereas the simulated tropopause is too low in the subtropical transition zone (Fig. 10a). With respect to the height of the vertical temperature minimum, the socalled cold point tropopause (CPT), the differences between both model versions show a similar signature as for the thermal tropopause height. Note that both height and temperature of the thermal tropopause as well as of the cold point tropopause are derived at every integration time step from the modeled temperature field and not from a monthly mean temperature distribution. The height of the cold point tropopause is defined as the respective model level with the vertical minimum temperature. Since the available ERA40 data have a reduced vertical resolution in the tropopause region compared to E39, the ERA40 cold point tropopause has not been determined.

In the tropics, the thermal as well as the cold point tropopause are significantly colder (2-3 K) in E39/ATTILA than in E39/SLT (Fig. 10b). However, as mentioned in Sect. 5.1, tropical tropopause temperatures are crucial for the stratospheric water content above $100 \mathrm{hPa}$ (sometimes called the "stratospheric overworld"). The so-called entry level mixing ratio is a measure for the water vapor mixing ratio of air parcels entering the stratosphere (Kley et al. 2000). The entry level can be derived from coincident water vapor and methane measurements or, as in this case, the average water vapor mixing ratio at the cold point tropopause between $20^{\circ} \mathrm{N}$ and $20^{\circ} \mathrm{S}$. As a consequence of the temperature decrease at the tropical tropopause, the entry level mixing ratio is reduced in E39/ATTILA compared to E39/SLT: The annual mean entry level amounts $4.4 \mathrm{ppmv}$ in E39/ATTILA and $6.3 \mathrm{ppmv}$ in E39/SLT, respectively. This difference explains the above mentioned reduction of the stratospheric water vapor mixing ratio in E39/ATTILA (Table 1, Fig. 3). Consistent with the reduced tropopause height in the extratropics, the 

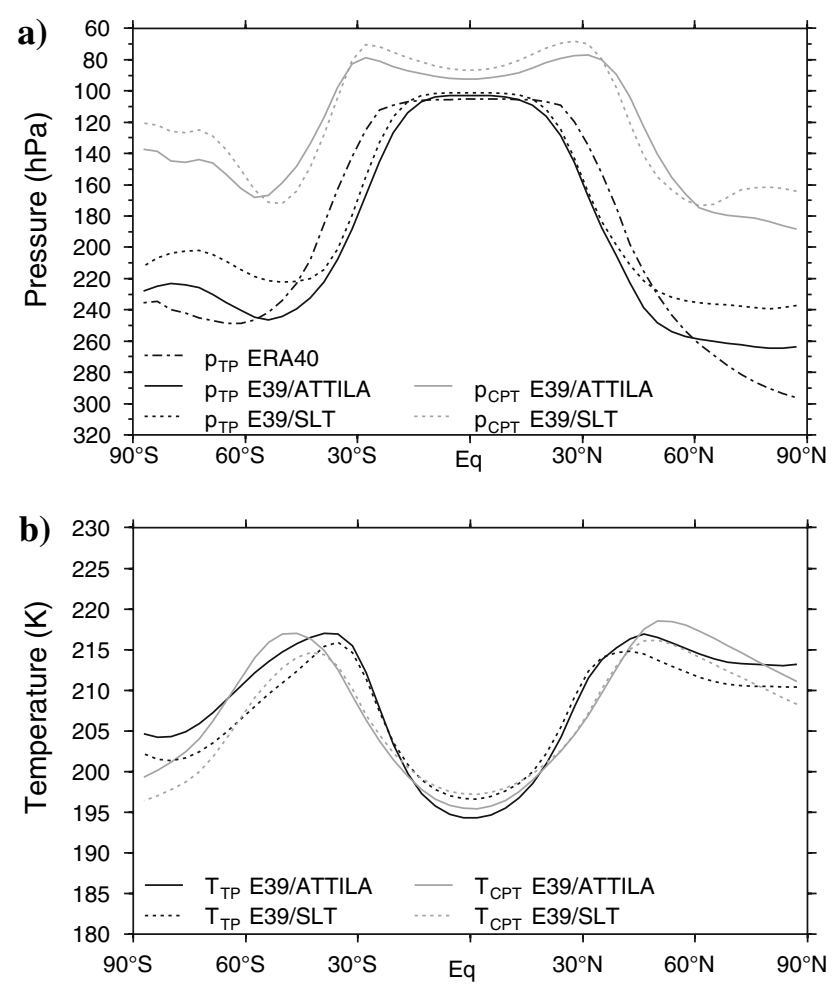

Fig. 10 Annual averages of zonal mean height (a) and temperature (b) of thermal tropopause (TP black) and cold point tropopause (CPT bold, grey) in E39/SLT (dotted lines) and E39/ATTILA (solid lines). The bold dashed dotted line indicates the annually averaged zonal mean height of the thermal tropopause derived from ERA40 data

tropopause temperatures are approximately $5 \mathrm{~K}$ higher in E39/ATTILA compared to E39/SLT.

\subsection{Zonal wind}

The above described improvements in the simulated temperature distribution result in some notable changes of the modeled circulation, which are presented in this subsection in terms of the zonal wind field.

Figure 11a displays the observed zonally averaged zonal wind field for January and July, based on ERA40 data. Climatologies of zonal mean wind fields as simulated with E39/SLT and E39/ATTILA are presented in Fig. 11b and c. Both model versions reproduce the main characteristics of the observed zonal wind field. The position of the subtropical and the polar night jets are in reasonable agreement with ERA40 data. A transition zone with reduced wind speed is modeled in both hemispheres, which separates subtropical and polar night jets. Results from different model intercomparison projects (Gates et al. 1999; Pawson et al. 2000) show that the separation between winter subtropical and polar night jet is a potential problem in GCMs, at least in northern hemispheric winter.
The largest deviations from ERA40 occur in the southern hemisphere during winter. Associated with the cold temperature bias in the polar lower stratosphere the polar vortex as simulated in E39/SLT is too strong by approximately $15 \mathrm{~m} \mathrm{~s}^{-1}$. The reduction of the simulated cold temperature bias in the southern polar stratosphere in E39/ ATTILA results in a weakening of the modeled polar vortex compared to E39/SLT, which means a better agreement with observations. However, the polar night jet in E39/ATTILA is still approximately $10 \mathrm{~m} \mathrm{~s}^{-1}$ stronger than observed. In the northern hemisphere, the polar night jet as simulated with both model versions is also slightly too strong compared to ERA40 (Table 2).

Another improvement in E39/ATTILA is found in the stratosphere during summer months. In the northern hemisphere, the calculated easterlies are too strong in both model versions. In the southern hemisphere, E39/SLT does not reproduce easterly winds between 20 and $100 \mathrm{hPa}$ at high latitudes. The correct simulation of stratospheric easterlies in the southern hemisphere is a widespread problem of atmospheric GCMs and seems to be independent of the height of the model top (Gates et al. 1999; Pawson et al. 2000). These model deficiencies might be linked to the simulated temperature bias in the polar stratosphere which results in a too stable polar vortex and a delayed breakdown of the vortex. Associated with the reduced cold pole temperature error (Sect.5.2) E39/ATTILA simulates easterlies in the southern stratosphere above the $30 \mathrm{hPa}$ level with slightly higher wind speeds than in E39/SLT.

In order to illustrate in more detail the progress concerning the modeled polar vortex dynamics, Fig. 12 presents the climatological mean annual cycle of zonal means of stratospheric zonal winds at $30 \mathrm{hPa}, 60^{\circ} \mathrm{N} / \mathrm{S}$ as simulated with E39/SLT (b) and E39/ATTILA (c). For comparison, analyses of observations from the National Centers for Environmental Prediction (NCEP, provided by P. Newman, E. Nash, and R. Nagatani, data accessible via "http://code916.gsfc.nasa.gov") are shown in the upper panel of Fig. 12. As a measure of the interannual variability, the minimum-to-maximum range of the zonal wind speed is shaded.

In the northern hemisphere, the mean zonal winds in mid-winter are weaker in E39/ATTILA than in E39/SLT (30 $\mathrm{m} \mathrm{s}^{-1}$ versus $35 \mathrm{~m} \mathrm{~s}^{-1}$ ). Furthermore, easterly wind speed in early July is slightly higher in E39/ATTILA than in E39/SLT ( $5 \mathrm{~m} \mathrm{~s}^{-1}$ versus $3 \mathrm{~m} \mathrm{~s}^{-1}$ ), which is both in better agreement NCEP analyses. The interannual variability as simulated in both models E39/SLT and E39/ ATTILA is comparable to observations, with minor and major stratospheric warmings frequently occurring. About nine minor warmings, which are associated with a clear decrease of the westerly wind speed and a reversal of the 
Fig. 11 Climatological averages of the zonal mean zonal wind speed $\left(\mathrm{m} \mathrm{s}^{-1}\right)$ for January and July: a ERA40, b E39/SLT, c E39/ATTILA. The contour interval in $5 \mathrm{~m} \mathrm{~s}^{-1}$, the bold line indicates the 0 -contour, negative contours are dashed. Wind speeds above $60 \mathrm{~m} \mathrm{~s}^{-1}$ are shaded a)

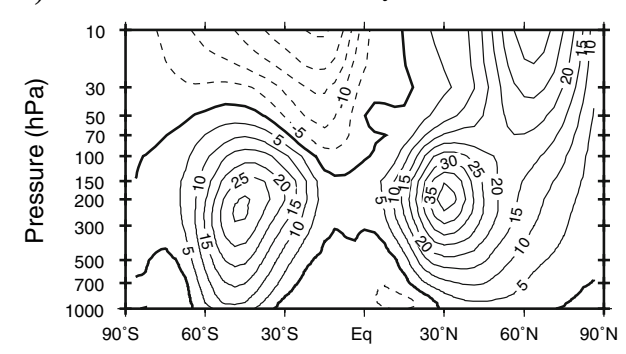

b)
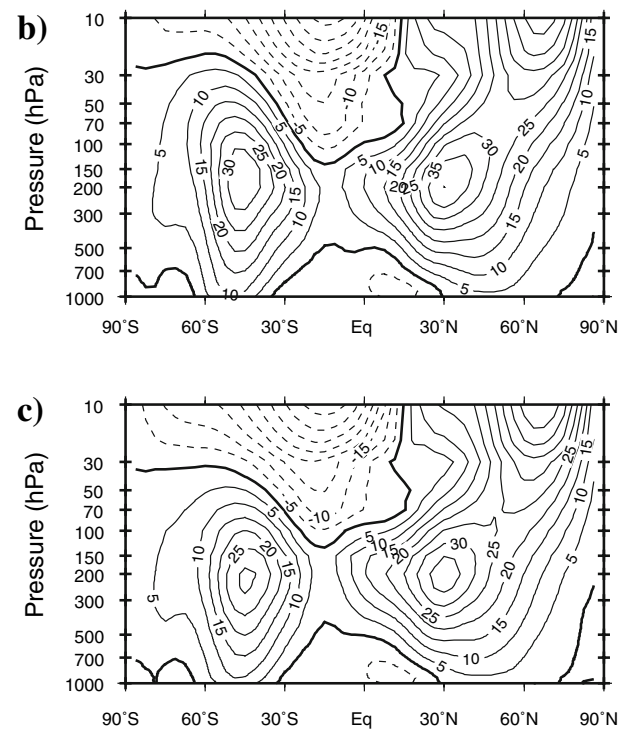

July
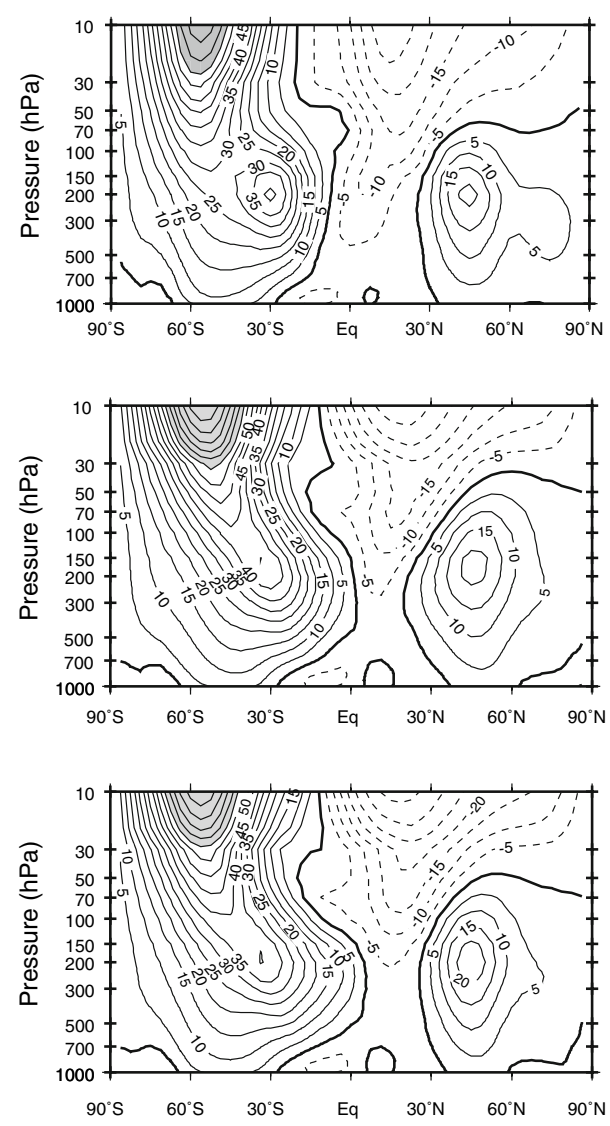

temperature gradient between polar latitudes and $60^{\circ} \mathrm{N}$, are simulated in both model versions E39/SLT and E39/ATTILA during 10 years of simulation. Additionally, 3 to 4 so-called strong minor warmings, i.e. a reduction of the westerly winds to less than $10 \mathrm{~m} \mathrm{~s}^{-1}$ and a reversal of the temperature gradient, are found.

In the southern hemisphere, the most important gain for E39/ATTILA is the simulated wind reversal in summer at $60^{\circ} \mathrm{S}, 30 \mathrm{hPa}$. However, the modeled easterly wind speed in early July peaks at only $1.7 \mathrm{~m} \mathrm{~s}^{-1}$, whereas the NCEP analyses show mean easterly wind speeds of approximately $7 \mathrm{~m} \mathrm{~s}^{-1}$. E39/SLT is generally not able to simulate the observed wind reversal in summer within this stratospheric region. Furthermore, the annual cycle of the zonal wind as simulated with E39/ATTILA is improved compared to E39/SLT. In E39/ATTILA the zonal wind speed increases steadily in autumn and winter and peaks at $60 \mathrm{~m} \mathrm{~s}^{-1}$ in early September, which in good agreement with the NCEP data. In contrast, E39/SLT shows slightly decreasing wind speeds at the end of June and nearly constant westerlies with values about $55 \mathrm{~m} \mathrm{~s}^{-1}$ in August and September. The interannual variability (in terms of peak-to-peak range) of southern hemisphere zonal winds is underestimated in both model versions, even more so in E39/ATTILA. This behavior is probably related to the simulated cold temperature bias near the model top which leads to a too stable Antarctic vortex (Table 2). It should be mentioned that the exceptional high variability of the NCEP data to be noted in September/October is caused by the 2002 Antarctic vortex split.

\section{Summary and discussion}

In this paper we applied the purely Lagrangian, numerically non-diffusive advection scheme ATTILA for the transport of water vapor and cloud water in the general circulation model E39, instead of the operational semiLagrangian transport scheme (SLT). The main motivation for this study was the assumed relationship between wet and cold bias in the extratropical lowermost stratosphere as simulated with E39/SLT, and the numerical properties of the respective advection schemes: Previous studies have shown that ATTILA is able to maintain steeper gradients than the semi-Lagrangian scheme which suffers from a considerable numerical diffusion (Reithmeier and Sausen 2002). The presented approach comprises a combination of Lagrangian advection and Eulerian parameterizations of physical processes which requires a transformation between both spaces. While the 
Fig. 12 Zonal means of the zonal wind speed $\left(\mathrm{m} \mathrm{s}^{-1}\right)$ at $30 \mathrm{hPa}, 60^{\circ} \mathrm{N}($ left $)$ and $60^{\circ} \mathrm{S}$ (right). Top NCEP analyses 1979-2004, Middle E39/SLT, Bottom E39/ATTILA. Shaded areas indicate the interannual variability (minimum to maximum)
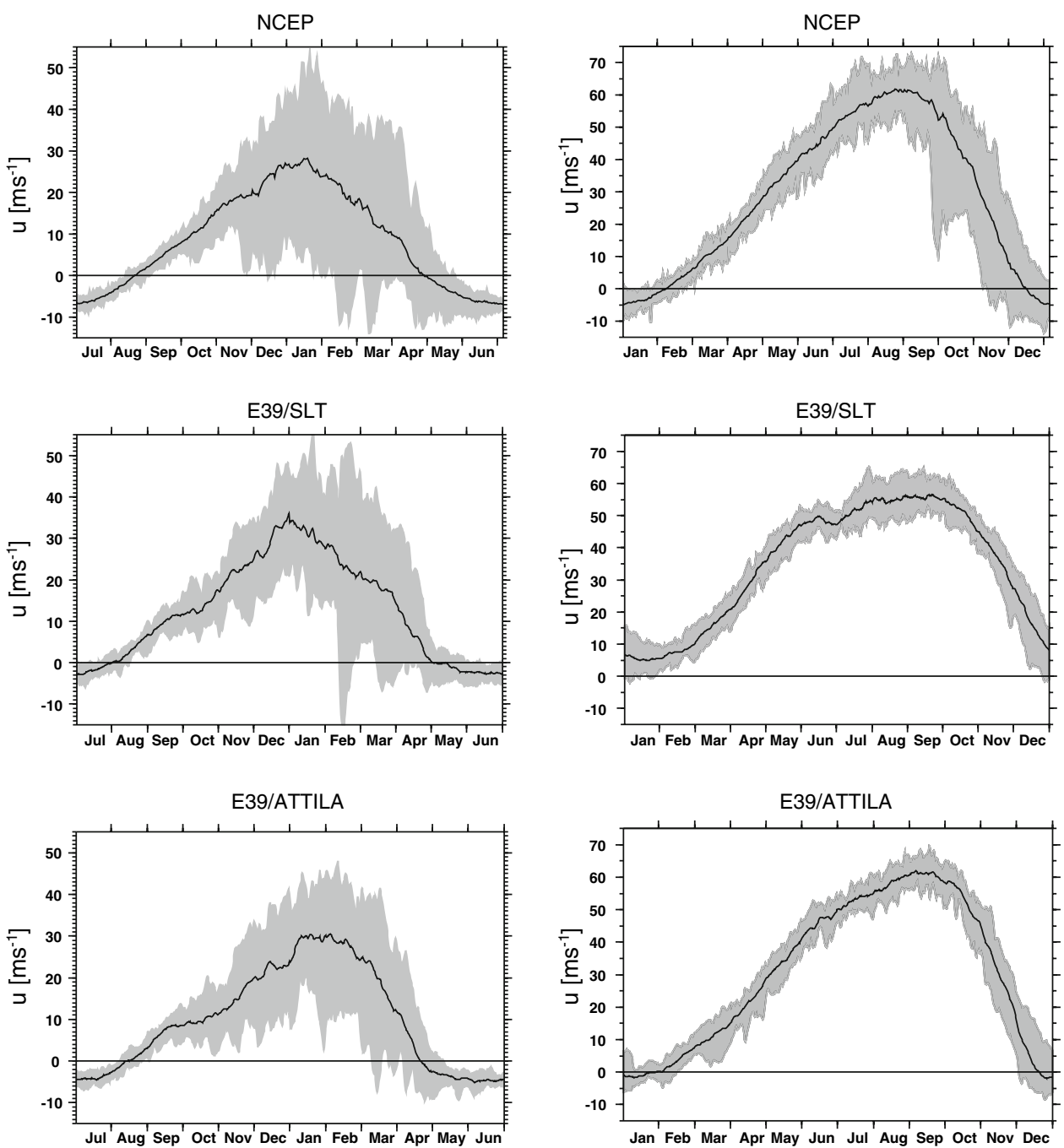

Lagrangian advection itself is perfectly mass conserving, inconsistencies between the Lagrangian and Eulerian density distribution lead to a global water vapor mass mismatch between both spaces of about $1 \%$. Since that error does not influence the transported mass, and since the hydrological cycle in ECHAM4 itself contains a small imbalance, the mentioned mass mismatch is negligible.

Compared to E39/SLT, E39/ATTILA shows a remarkably reduced water vapor content in the extratropical lowermost stratosphere as well as a steeper meridional gradient in the subtropics, resulting in a much better agreement with observations. A sensitivity study with E39/ ATTILA in which an idealized "water vapor" tracer was advected by the original semi-Lagrangian scheme confirms that the strong horizontal diffusion of water vapor from the tropical upper troposphere into the extratropical lowermost stratosphere is the main contributor to the simulated wet bias in E39/SLT.

A comparison of the zonal mean temperature distribution as simulated with E39/SLT and E39/ATTILA, respectively, indicate a significant improvement in modeled temperatures within the extratropical lowermost stratosphere. In E39/ATTILA the pronounced cold bias of $7 \mathrm{~K}$ and more, like in E39/SLT, is no longer apparent, and the simulated temperatures are much closer to ERA40 data. Sensitivity studies in which the model's radiation scheme was fed with a HALOE water vapor climatology confirm the conclusion that half of the cold bias in the extratropical tropopause region in E39/SLT is radiatively forced by the simulated wet bias. Furthermore, these temperature changes result in an improved representation of the tropopause, especially in the extratropics. Temperatures at the tropical tropopause are slightly lower in E39/ATTILA which leads to a favorable overall reduction of stratospheric water vapor mixing ratios.

The cold bias problem has often been considered as being typical for GCMs with a model top at $10 \mathrm{hPa}$, although the cold bias is also present in a number of middle atmosphere GCMs (Pawson et al. 2000). This study has shown, that-at least in the E39 GCM-more than half of the simulated cold 
bias can be related to the simulated wet bias. One possible reason for the remaining cold bias might be an inappropriate spatial model resolution. A study of Roeckner et al. (2006) using the ECHAM5 GCM showed that increasing the vertical resolution from 19 to 31 model layers may lead to a reduction of the simulated cold bias in the extratropical tropopause region of up to $50 \%$. This result is in disagreement with a study of Land et al. (1999) who showed that an increased vertical resolution from 19 to 39 levels does hardly affect the cold bias in the ECHAM4 GCM. However, Roeckner et al. (2006) used models of much higher horizontal resolution (from T42 up to T106), pointing out the necessity of increasing horizontal and vertical resolution at the same time to exploit the dynamic advantage of a resolution increase. Hence, in the simulations of Roeckner et al. (2006) a reduced numerical diffusion due to enhanced resolution and improvements of the model dynamics work simultaneously. One might speculate that in their models the remaining cold/wet bias is due to the remaining diffusivity of the advection scheme, while in our simulation it is due to the dynamic deficits arising from a coarse horizontal resolution. Beside a too strong longwave emission due to the wet bias, a too weak shortwave absorption of solar radiation by e.g. ozone might contribute to the simulated temperature bias (Pawson et al. 2000). A further possible explanation was given by Johnson (1997) who stated that the simulated cold bias in the extratropical tropopause region is a consequence of aphysical sources of entropy like numerical diffusion, Gibbs oscillations or inadequate parameterizations. Overall the discussion about the remaining cold bias is rather speculative, particularly as the reasons might differ between different models.

The simulated temperature errors in E39/SLT are found to be responsible for some deficiencies in model dynamics. As mentioned above, the cold bias in the extratropical tropopause region affects the location of and the temperature at the tropopause which causes some further problems, e.g. concerning the simulated volume of tropospheric and stratospheric air or the radiative forcing of greenhouse gases in the tropopause region. Furthermore, systematically low temperatures in the southern hemisphere polar vortex (cold pole) influence modeled stratospheric dynamics. In the southern stratosphere, E39/ATTILA shows a weaker polar night jet than E39/SLT and a wind reversal in upper model levels at polar latitudes during summer which is not apparent in E39/SLT. Both changes result in a better agreement with observations.

Finally, the cold bias leads to problems concerning the parameterization of cirrus clouds and the formation of contrails. The above mentioned shortcomings might affect model studies of the climate impact of aviation or climate change projections in general. Therefore, the transport of water vapor and cloud water with the Lagrangian advection scheme ATTILA does not only constitute a major improvement compared to E39/SLT with respect to modeled water vapor and temperatures, but is a significant contribution to climate modeling in general.

With respect to the fully coupled chemistry-climate model ECHAM4.L39(DLR)/CHEM (E39/C), Hein et al. (2001) and Dameris et al. (2005) reported a too cold and too stable southern hemisphere polar vortex with a delayed vortex breakdown. This model behavior has a direct impact on Antarctic ozone chemistry and the simulation of the ozone hole which persists too long into spring. Additionally, the cold bias might result in enhanced formation of polar stratospheric clouds and hence in unrealistic ozone destruction in the lowermost stratosphere. This shortcoming does not only affect the modeled ozone distribution, but might also affect model predictions concerning the development of the ozone layer in a changing climate. Therefore, the Lagrangian transport of water vapor and cloud water should also improve some aspects of modeled ozone chemistry.

Furthermore, ATTILA allows the transport of a large number of tracers without exceeding reasonable limits of computing time, which is an additional advantage in case of many chemical species or many classes of aerosols. Based on the results of the present study we conclude that ATTILA is not restricted to passive tracers only, but might be also successfully applied for the transport of species that feed back to dynamics. First results with the interactively coupled chemistry-climate model E39/C using ATTILA for the transport of all trace species look quite promising and will be published separately.

Acknowledgments We would like to thank C. Reithmeier for help with implementing the Lagrangian transport of water vapor and cloud water in ATTILA. M. Dameris and R. Sausen are kindly acknowledged for their constructive suggestions and many helpful discussions. We are also grateful to S. Pechtl for assistance with the re-adjustment of cloud parameters in the model version E39/ATTILA and J.-U. Grooß for providing the HALOE water vapor climatology. Four anonymous referees are also kindly acknowledged for their helpful comments. Part of this work was funded by the COBI project within a national climate research program (DEKLIM) of the German Federal Ministry of Education and Research (BMBF). ECMWF ERA40 data used in this study have been obtained from the ECMWF data server. The GPCP combined precipitation data were developed and computed by the NASA/Goddard Space Flight Center's Laboratory for Atmospheres as a contribution to the GEWEX Global Precipitation Climatology Project. The model simulations have been performed on the NEC SX-6 high performance computer of the German Climate Computing Center (DKRZ), Hamburg.

\section{References}

Adler RF, Huffman GJ, Chang A, Ferraro R, Xie P, Janowiak J, Rudolf B, Schneider U, Curtis S, Bolvin D, Gruber A, Susskind J, Arkin P (2003) The version 2 global precipitation climatology 
project (GPCP) monthly precipitation analysis (1979-present). J Hydrometeor 4:1147-1167

Brinkop S, Sausen R (1997) A modified mass-flux scheme for convection which maintains positive tracer concentrations. Beitr Phys Atmos 70:245-248

Clough SA, Iacono MJ, Moncet J-L (1992) Line-by-line calculations of atmospheric fluxes and cooling rates: application to water vapor. J Geophys Res 97:15761-15785

Dameris M, Grewe V, Ponater M, Eyring V, Mager F, Matthes S, Schnadt C, Stenke A, Steil B, Brühl C, Giorgetta M (2005) Long-term changes in a transient simulation employing an interactively coupled chemistry-climate model under realistic forcings. Atmos Chem Phys 5:2121-2145

Fels SB, Mahlman JD, Schwarzkopf MD, Sinclair RW (1980) Stratospheric sensitivity to perturbations in ozone and carbon dioxide: radiative and dynamical response. J Atmos Sci 37:2265-2297

Gates WL, Boyle JS, Covey C, Dease CG, Doutriaux CM, Drach RS, Fiorino M, Glecker PJ, Hnilo JJ, Marlais SM, Phillips TJ, Potter GL, Santer BD, Sperber KR, Taylor KE, Williams DN (1999) An overview of the results of the atmospheric model intercomparison project (AMIP I). Bull Am Meteor Soc 80:29-55

Grewe V, Dameris M, Fichter C, Sausen R (2002) Impact of aircraft $\mathrm{NO}_{\mathrm{x}}$ emissions. Part 1: Interactively coupled climate-chemistry simulations and sensitivities to climate-chemistry feedback, lightning and model resolution. Meteorology Z 3:177-186

Grooß J-U, Russell III JM (2005) Technical note: a stratospheric climatology for $\mathrm{O}_{3}, \mathrm{H}_{2} \mathrm{O}, \mathrm{CH}_{4}, \mathrm{NO}_{\mathrm{x}}, \mathrm{HCl}$ and $\mathrm{HF}$ derived from HALOE measurements. Atmos Chem Phys 5:2797-2807

Hein R, Dameris M, Schnadt C, Land C, Grewe V, Köhler I, Ponater M, Sausen R, Steil B, Landgraf J, Brühl C (2001) Results of an interactively coupled atmospheric chemistry-general circulation model: Comparison with oberservations. Ann Geophys 19: $435-457$

Heymsfield AJ (1977) Precipitation development in stratiform clouds. A microphysical and dynamical study. J Atmos Sci 34:367-381

Jöckel P, Sander R, Kerkweg A, Tost H, Lelieveld J (2005) Technical note: the modular earth submodel system (MESSy)—a new approach towards earth system modeling. Atmos Chem Phys 5:433-444

Johnson DR (1997) "General coldness of climate models" and the second law: implications for modeling the earth system. J Clim 10:2816-2846

Kley D, Russell III JM, Phillips C (2000) SPARC Assessment of upper tropospheric and stratospheric water vapour, WCRP \#113, WMO/TD \#1043, SPARC Report \#2

Land C, Ponater M, Sausen R, Roeckner E (1999) The ECHAM4.L39(DLR) atmosphere GCM-Technical description and model climatology, DLR Forschungsbericht 1999-31, ISSN 1434-8454, Köln. http://www.pa.op.dlr.de/echam/L39.html

Land C, Feichter J, Sausen R (2002) Impact of vertical resolution on the transport of passive tracers in the ECHAM4 model. Tellus (B) $54: 344-360$

Mote PW, Holton JR, Boville BA (1994) Characteristics of stratosphere-troposphere exchange in a general circulation model. J Geophys Res 99:16815-16829

Pawson S, Kodera K, Hamilton K, Shepherd TG, Beagley SR, Boville B, Farrara JD, Fairlie TDA, Kitoh A, Lahoz WA, Langematz U, Manzini E, Rind DH, Scaife AA, Shibata K, Simon P, Swinbank R, Takacs L, Wilson R, Al-Saadi JA, Amodei M, Chiba M, Coy L, de Grandpre J, Eckman RS, Fiorino M, Grose WL, Koide H, Koshyk JN, Li D, Lerner J, Mahlman JD, McFarlane NA, Mechoso CR, Molod A, O'Neill A, Pierce RB, Randel WJ, Rood $\mathrm{RB}, \mathrm{Wu} F$ (2000) The GCM-reality inter-comparison project of SPARC (GRIPS): scientific issues and initial results. Bull Am Meteorol Soc 81:781-796
Rasch PJ, Lawrence M (1998) Recent development in transport methods at NCAR, Report No. 265, Max-Planck-Institut für Meteorologie, Hamburg

Rasch PJ, Williamson DL (1990) Computational aspects of moisture transport in global models of the atmosphere. Q J R Meteorol Soc 116:1071-1090

Reichler T, Dameris M, Sausen R (2003) Determining the tropopause height from gridded data. Geophys Res Lett 30:2042, doi: 10.1029/2003GL018240

Reithmeier C, Sausen R (2002) ATTILA: atmospheric tracer transport in a Lagrangian model. Tellus (B) 54:278-299

Roeckner E, Arpe K, Bengtsson L, Christoph M, Claussen M, Dümenil L, Esch M, Giorgetta M, Schlese U, Schulzweida U (1996) The atmospheric general circulation model ECHAM-4: model description and simulation of present-day climate, Report No. 218, Max-Planck-Institut für Meteorologie, Hamburg

Roeckner E, Bäuml G, Bonaventura L, Brokopf R, Esch M, Giorgetta M, Hagemann S, Kirchner I, Kornblueh L, Manzini E, Rhodin A, Schlese U, Schulzweida U, Tompkins A (2003) The atmospheric general circulation model ECHAM-5. Part I: Model description, Report No. 349, Max-Planck-Institut für Meteorologie, Hamburg

Roeckner E, Brokopf R, Esch M, Giorgetta M, Hagemann S, Kornblueh L, Manzini E, Schlese U, Schulzweida U (2006) Sensitivity of simulated climate to horizontal and vertical resolution in the ECHAM5 atmosphere model. J Clim 19:3771-3791

Rosier SM, Shine KP (2000) The effect of two decades of ozone change on stratospheric temperature as indicated by a general circulation model. Geophys Res Lett 27:2617-2620

Smagorinsky J (1963) General circulation experiments with the primitive equations. Mon Wea Rev 91:99-164

Stendel M, Roeckner E (1998) Impacts of horizontal resolution on simulated climate statistics in ECHAM4, Report No. 263, MaxPlanck-Institut für Meteorologie, Hamburg

Stenke A, Grewe V (2005) Simulation of stratospheric water vapor trends: impact on stratospheric ozone. Atmos Chem Phys 5:1257-1272

Stuber N, Sausen R, Ponater M (2001) Stratosphere adjusted radiative forcing calculations in a comprehensive climate model. Theor Appl Climatol 68:125-135

Tiedtke M (1989) A comprehensive mass flux scheme for cumulus parameterization in large-scale models. Mon Wea Rev 117:1779-1800

Timmreck C, Graf H-F, Feichter J (1999) Simulation of Mt. Pinatubo Volcanic aerosol with the Hamburg climate model ECHAM4. Theor Appl Climatol 62:85-108

Uppala SM, Kållberg PW, Simmons AJ, Andrae U, Da Costa Bechtold V, Fiorino M, Gibson JK, Haseler J, Hernandez A, Kelly GA, Li X, K O, Saarinen S, Sokka N, Allan RP, Andersson E, Arpe K, Balmaseda MA, Beljaars ACM, van de Berg L, Bidlot J, Bormann N, Caires S, Chevallier F, Dethof A, Dragosavac M, Fisher M, Fuentes M, Hagemann S, Hólm E, Hoskins BJ, Isaksen L, Janssen PAEM, Jenne R, McNally AP, Mahfouf J-F, Morcrette J-J, Rayner NA, Saunders RW, Simon P, Sterl A, Trenberth KE, Untch A, Vasiljevic D, Viterbo P, Woollen J (2005) The ERA-40 re-analysis. Q J R Meteorol Soc 131:2961-3012

Walton JJ, MacCracken MC, Ghan SJ (1988) A global-scale Lagrangian trace species model of transport, transformation, and removal processes. J Geophys Res 93:8339-8354

Williamson DL, Rasch PJ (1994) Water vapor transport in the NCAR CCM2. Tellus (A) 46:34-51

Xu KM, Krüger SK (1991) Evaluation of cloudiness parameterizations using a cumulus ensemble model. Mon Wea Rev 119: 342-367 